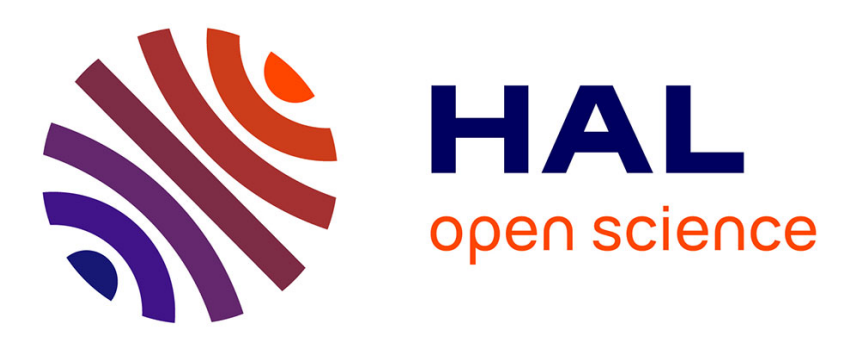

\title{
Image-based evaluation of treatment responses of facial wrinkles using LDDMM registration and Gabor features
}

\author{
Nazre Batool, Josiane Zerubia
}

\section{To cite this version:}

Nazre Batool, Josiane Zerubia. Image-based evaluation of treatment responses of facial wrinkles using LDDMM registration and Gabor features. ICIP 2015 - IEEE International Conference on Image Processing, Sep 2015, Québec, Canada. hal-01154005

\section{HAL Id: hal-01154005 \\ https://hal.inria.fr/hal-01154005}

Submitted on 21 May 2015

HAL is a multi-disciplinary open access archive for the deposit and dissemination of scientific research documents, whether they are published or not. The documents may come from teaching and research institutions in France or abroad, or from public or private research centers.
L'archive ouverte pluridisciplinaire HAL, est destinée au dépôt et à la diffusion de documents scientifiques de niveau recherche, publiés ou non, émanant des établissements d'enseignement et de recherche français ou étrangers, des laboratoires publics ou privés. 


\title{
IMAGE-BASED EVALUATION OF TREATMENT RESPONSES OF FACIAL WRINKLES USING LDDMM REGISTRATION AND GABOR FEATURES
}

\author{
Nazre Batool Josiane Zerubia \\ INRIA, Team AYIN, 2004 route des Lucioles, 06902 Sophia Antipolis Cedex, France \\ email: firstname.lastname@inria.fr
}

\begin{abstract}
This paper presents image-based quantitative evaluation of subtle variations in facial wrinkles for the same subject in response to a dermatological treatment. This is a novel application because the time series images of the same subject over a shorter time period of weeks are analyzed as compared to more prevalent inter-person analysis of facial skin/marks. We propose image features based on Gabor filter bank for an accurate quantitative evaluation of variations in facial wrinkles. Since variations in Gabor features are very small on a time period of weeks, we propose a framework to compare image features in key wrinkle sites only while excluding the noise introduced by non-wrinkle sites. The framework consists of finer registration of images using Large Deformation Diffeomorphic Metric Mapping (LDDMM) and detection of wrinkle sites using Gabor filter bank and morphological image processing. Preliminary experiments show that the framework is useful in calculating variations in Gabor features at detected sites and indicating trends in the response of facial wrinkles to the dermatological treatment.
\end{abstract}

Index Terms- Face image analysis, Skin texture, Detection of wrinkles, Response to treatment, LDDMM registration, Gabor filters, Morphological processing

\section{INTRODUCTION}

In recent years, image-based analysis of atypical facial features (marks, scars, moles, wrinkles, etc.) has been done for a variety of applications e.g. facial marks for recognition between twins [1], evaluation of severity of wrinkles [2,3], inpainting of marks/wrinkles [4], localization of wrinkles [5, 6], segmentation of acne scars [7], synthesis of aging skin texture for aging simulations $[8,9]$. Analysis of facial features can be done in images of different individuals or those of the same individual taken at different times. However, to the best of our knowledge, the problem of analyzing minute differences in facial features in images of the same individual remains unaddressed. Such an analysis can aid several applications; for example, temporal changes in facial texture, geometry and wrinkles can indicate the unique aging pattern of a person and changes in moles, brown spots, acne can indicate the efficacy of a dermatological treatment. The detection of variations occurring over shorter periods of time, say weeks or months, is more involved than that of the variations happening over years. In this paper, we present a quantitative evaluation of changes in facial wrinkles around eyes, called lateral canthal lines/crow's feet, in response to a dermatological procedure of 'botulinum toxin' injections. The closest to our work is Cula's work [2,3] where automatic detection and scoring of facial wrinkles were done using the orientation and frequency of the elongated spatial features captured via Gabor filters. Our work is different in the aspect that we conduct comparative analysis of the images of the same subject taken over a period of 52 weeks. Furthermore, we use blue channel in RGB images as skin marks caused by wrinkles, brown spots, freckles, etc. are more prominent in blue channel due to the specific physical properties of skin. Such skin features appearing in blue channel as blobs/lines aid in the registration of skin images.

For quantitative evaluation, we calculate responses to $\mathrm{Ga}-$ bor filter banks in two ways: (a) in the whole skin patch around eyes and (b) a wrinkle template created from the automatic detection of wrinkles in that skin patch. We hypothesize that the results based on image features obtained from the latter method are more accurate as the interference caused by image features in non-wrinkle sites are excluded. The dataset for this work was provided by a world leader company in dermatology where images were taken in controlled lighting and pose settings. The severity of crow's feet before and after the treatment was scored clinically according to an established dermatological scoring method $[10,11]$ as numbers $\{0,1,2,3\}$ in the order of increasing severity. A common approach to monitoring changes in biological images is the registration of images to an atlas where areas of movement during registration represent temporal changes. In the absence of an atlas we adopt a 2-step approach of registration and quantitative evaluation. For accuracy, fine registration of images to the level of wrinkles is required due to slight pose variations. Fig. 1(a) shows the baseline image of a subject with the location of 4 land marks marked as red dots. Fig. 1(b) shows a later image registered using projective similarity to the baseline. In Fig. 1(c) misalignment in wrinkles can be observed even after registration. In a typical face recognition 


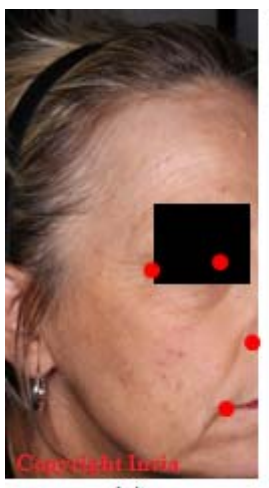

(a)

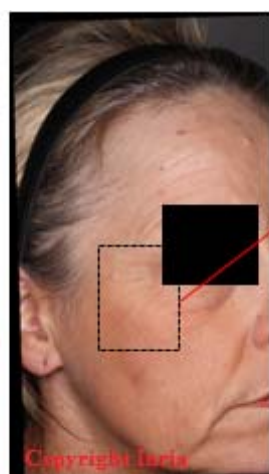

(b)

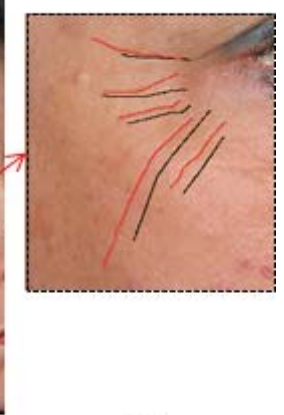

(c)
Fig. 1. A simple registration scheme based on land marks does not register wrinkles. (a) Baseline with land marks shown as red dots. (b) Week 4 image after registration. (c) Misaligned wrinkles after registration.

system, such a slight pose variation would be trivial but offers a challenge in our case when calculating subtle variations in Gabor responses in key wrinkle sites. We propose Large Deformation Diffeomorphic Metric Mapping (LDDMM) algorithm to achieve finer registration and then use the first author's previously proposed algorithm [6] to detect key wrinkle sites. Although an intuitive approach would be to detect and compare changes in the structural properties of wrinkles such as length, width or depth, however, such an approach would require prior knowledge about wrinkle locations either provided by a user or detected automatically. Drawing wrinkles on facial images is tedious and time consuming whereas the image-based detection of wrinkles is based on image features. Hence, in this work we focus on image features (Gabor features) and leave the analysis of the structural properties of automatically detected wrinkles for later work. Our main contribution is: (a) this work is an initial attempt at analyzing subtle variations in facial wrinkles for the same individual and (b) we propose to compare Gabor responses in key wrinkle sites in registered images for more accurate quantitative evaluation.

\section{APPROACH}

\subsection{Data Setup}

The subjects in the dataset were Caucasian, male and female, 18 to 65 years of age with mild to severe crow's feet. Color images were taken in standard light modality with neutral facial expressions and were of size 1001 x 667 pixels. For each subject, baseline images were taken before the treatment and monitoring images were taken until 52 weeks after the treatment. The only pre-processing step was to register time series images to the baseline image for a subject using projective transformation in MATLAB ${ }^{\mathrm{TM}}$. Then user-provided
ROIs were used to extract skin patches around eyes. Let $I_{B}$ and $I_{n}$ denote blue channel images where $B$ denotes the baseline image and $n$ denotes the image taken at week $n$ where $n=\{4,8,12,16,24,36,48,52\}$. These images were registered on a finer scale to the baseline image using LDDMM as described next.

\subsection{Registration using Stationary LDDMM}

We approach the problem of finer registration as finding the appropriate deformation of underlying 2D image space. The method of surface deformation has been often used in medical imaging for registration in 2D or 3D spaces (for a survey on registration using deformation techniques please see [12]). Diffeomorphic transformations are invertible, preserve topology and both the function and its inverse are differentiable. We use the framework known as large deformation diffeomorphic metric mapping (LDDMM) proposed by Beg et al. [13] to study anatomical variability. The LDDMM framework was extended to solve landmark matching [14] and curve-matching $[15,16]$ for image registration. We do not use the LDDMM algorithm based on detected curves for wrinkles because (a) detected wrinkle curves can change from one image to another due to the treatment (b) skin texture features in blue channel images instead of just wrinkle curves in binary images can provide more comprehensive vector flows in LDDMM. Furthermore, LDDMM based on stationary velocity field was used rather than other LDDMM algorithms (e.g. [17]) due to the transformation smoothness which was recommended in a comparative study by Hernandez et al. [18].

We assume that wrinkles and other skin texture features are prominent enough to direct the vector field flow of LDDMM without any prior knowledge of correspondences between two images. The LDDMM tries to find a deformation (differentiable map $\varphi: \Omega \rightarrow \Omega$ with differentiable inverse map) of a compact space $\Omega$ (2D image space in our case). We refer the interested reader to [19] for mathematical and numerical implementation details of LDDMM registration based on image intensity and using stationary velocity fields. In stationary LDDMM, paths of diffeomorphisms $\varphi$ are parametrized by constant-time flows of vector fields $w$ belonging to the tangent space $V$ where the diffeomorphism can be computed from the group exponential map as follows:

$$
\varphi=\operatorname{Exp}(w) ; w \in V
$$

The problem of inexact matching between two images is solved by minimizing the following cost function:

$$
\mathrm{E}_{I_{n} \rightarrow I_{B}}=\|w\|_{V}^{2}+\frac{2}{\sigma^{2}}\left\|I_{n} \circ \varphi^{-1}-I_{B}\right\|_{\mathcal{L}^{2}}^{2}
$$

where the second term is a norm-squared cost measuring the degree of matching between two images and the first term is 


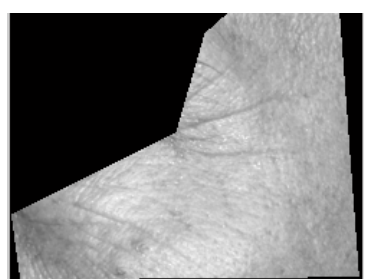

(a)

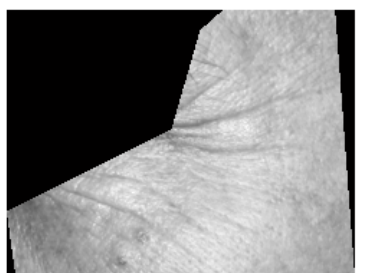

(b)

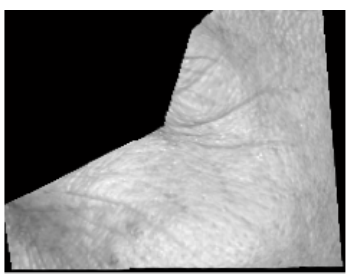

(c)

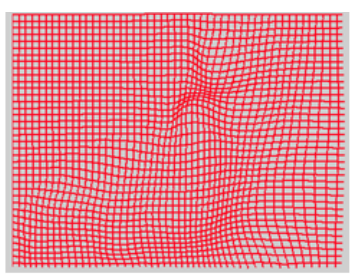

(d)

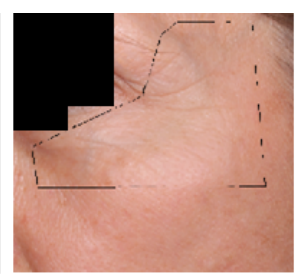

(e)

Fig. 2. Registration using LDDMM. (a) Source image (b) Target baseline image (c) Registered source image (d) Deformation of underlying 2D grid (e) Registered image shown in ROI.

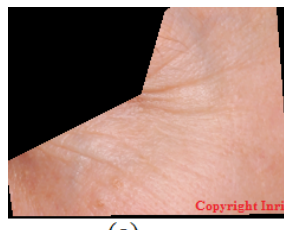

(a)

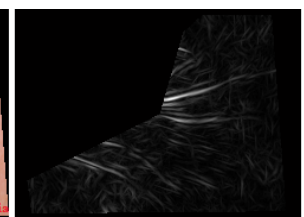

(b)

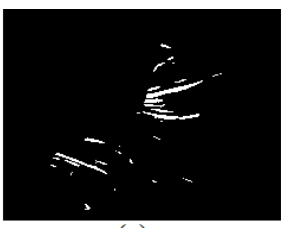

(c)

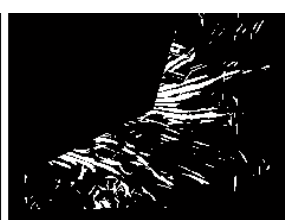

(d)

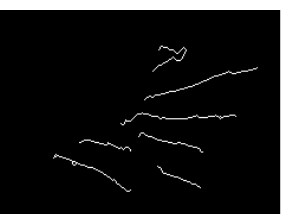

(e)

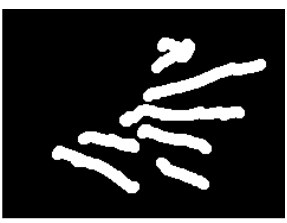

(f)

Fig. 3. Formation of image template. (a) Input image. (b) Gabor features. (c) Gabor features with a higher threshold value. (d) Gabor features with a lower threshold value. (e) Output of wrinkle detection algorithm. (f) Detected wrinkles dilated with a circular disc.

the regularization energy defined as a norm in space $V$ :

$$
<a, b>_{V} \doteq<\mathcal{L} a, \mathcal{L} b>_{\mathcal{L}^{2}}=<\mathcal{L}^{\dagger} \mathcal{L} a, b>_{\mathcal{L}^{2}}
$$

As in Beg's work [13], we use the diffusive model $\mathcal{L}=I d-$ $\alpha \nabla^{2}$ and Fourier domain to calculate the linear operator $K=$ $\left(\mathcal{L}^{\dagger} \mathcal{L}\right)^{-1}$ to calculate $\mathcal{L}^{2}$ norm in $V$ space. The gradient descent algorithm is used to optimize cost function with the gradient defined as:

$$
\nabla_{w} \mathrm{E}=2 w-\frac{2\left(\mathcal{L}^{\dagger} \mathcal{L}\right)^{-1}}{\sigma^{2}}\left(I_{n} \circ \varphi^{-1}-I_{B}\right)\left(\nabla I_{n} \circ \varphi^{-1}\right)
$$

and velocity $w$ updates are given as:

$$
w^{(k+1)}=w^{k}-\epsilon \nabla_{w} \mathrm{E}
$$

The algorithm initializes with $w=0 \in V$ and $\varphi=I d$. In gradient descent algorithm 40-50 iterations were sufficient and values of $\{\epsilon, \sigma, \alpha\}$ were set to be $\left\{5 \times 10^{-6}, 0.1,15\right\}$ for gray scale images in the range of [0-255]. Fig. 2 shows an example of registration using LDDMM.

\subsection{Detection of Key Wrinkle Sites using Gabor Features and Geometric Constraints}

Image features based on texture/appearance of image patches (e.g. Local Binary Patterns, Active Appearance Model features) can distinguish rougher aged skin texture and have been used in age estimation, recognition across aging and age progression [20]. However, skin texture discontinuities/cracks caused by wrinkles create image intensity gradients which are highlighted better by spatial filtering. Previously, spatial filtering has been used for detection and assessment of facial wrinkles e.g. Laplacian of Gaussian filters [21], Gabor filters banks [2, 3, 4, 6] and steerable filters [5]. Following the work of the first author in [4, 6], we use maximum Gabor filter amplitude responses at image sites as image features. Then we detect key wrinkle sites using an algorithm previously proposed by the first author in [6]. The algorithm is based on 'scaled' maximum Gabor filter responses and the incorporation of geometric constraints via morphological image processing (for detailed description of the algorithm please see [6]). The output of the algorithm is a binary image depicting detected key wrinkle sites. A template was created for each subject from the detected key wrinkle sites in that subject's baseline image by dilating the output binary image of the algorithm with a circular disk. As an example, Fig. 3 shows an image, detected wrinkles and dilated binary template. Fig. 3(c) and (d) show results of thresholding Gabor responses with two threshold values which result in under or over segmentation of wrinkle sites. Hence a simple thresholding of Gabor features is not considered to detect key wrinkle sites. The values used for Gabor and morphological parameters of the algorithm are given in Table 1 where $\sigma_{x}$ denotes the scale of the 2D Gaussian envelope, $f$ denotes the frequency of Gabor sinusoid and $\gamma$ denote the spatial aspect ratio of $\mathrm{Ga}-$ bor filter. Parameters $r^{d}, r_{N}, r_{E}, \theta_{N}, \theta_{E}$ denote the radii and angles of the disks used for dilation in the morphological processing and parameters $t_{L}, \Delta_{t}$ are the threshold values.

\section{RESULTS}

Fig. 4 includes a plot of results for five subjects where $\mathrm{x}$-axis represents monitoring time in number of weeks after the ad- 


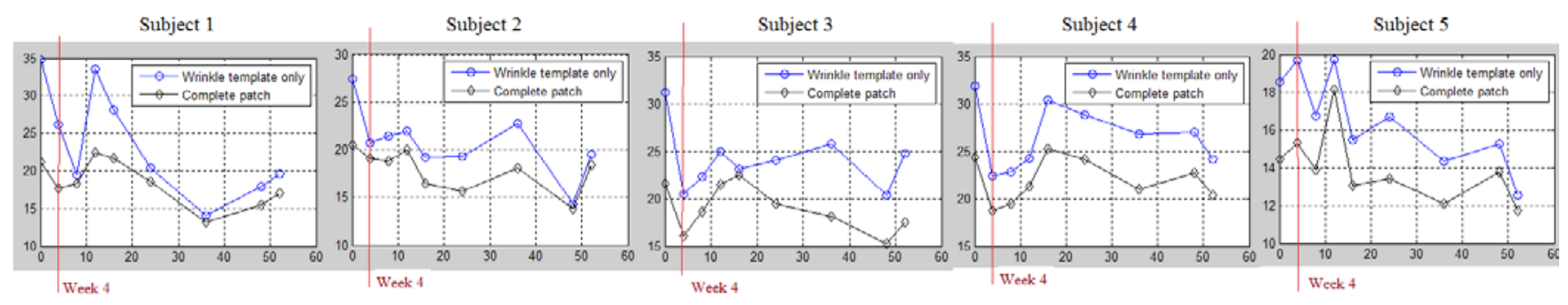

Fig. 4. Plot of results for five subjects where $x$-axis denotes number of weeks after the administration of treatment and $y$-axis denotes the average maximum Gabor response.

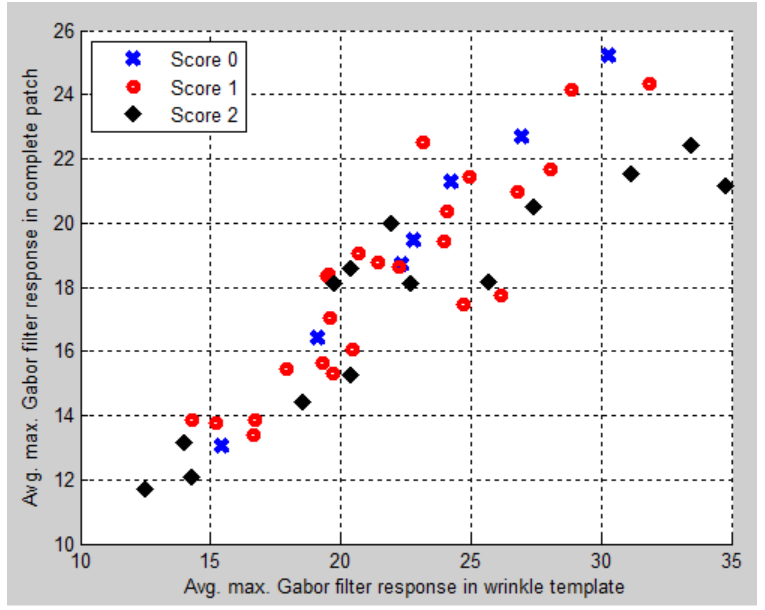

Fig. 5. Plot of Gabor features vs. clinical scores.

\begin{tabular}{|c|c|c|c|}
\hline Parameter & $f$ & $\sigma_{x_{1}}=\sigma_{x_{2}}$ & $\gamma$ \\
\hline Value & 1 & 0.5 & 15 \\
\hline \hline Parameter & $r^{d}$ (pixels) & $r_{N}$ (pixels) & $r_{E}$ (pixels) \\
\hline Value & 10 & 3 & $r_{N}-1$ \\
\hline \hline Parameter & $\theta_{N}=\theta_{E}$ & $t_{L}$ & $\Delta_{t}$ \\
\hline Value & $\frac{\pi}{3}$ & 0.15 & 0.1 \\
\hline
\end{tabular}

Table 1. Parameters for the detection algorithm in [6].

ministration of the treatment and y-axis represents the average of the maximum Gabor amplitude responses calculated in detected key wrinkle sites/template only (shown in blue) and in the whole skin patch (shown in black). It can be seen that both wrinkle templates and whole skin patches depict similar trends in subtle variations in Gabor responses. However, the variations in Gabor responses calculated in wrinkle templates are slightly larger than those calculated in whole skin patches. This verifies our initial assumption that subtle changes in wrinkles can be highlighted more by incorporating image features in wrinkle sites only instead of including both wrinkle and non-wrinkle sites. It can also be noted that Gabor responses drop significantly for most subjects (except subject 5) after the administration of the treatment in week
4. This is attributed to the lightening of wrinkles after the treatment which reduces resulting intensity gradients in images and hence Gabor amplitude responses. Fig. 5 shows a plot of average maximum Gabor amplitude response in key wrinkle sites and in the whole skin patches along with clinical scores for the five subjects over the period of 52 weeks where low correlation between Gabor responses and clinical scores can be observed. Hence, we conclude that although the present framework can capture subtle variations in facial wrinkles via Gabor features, it does not produce high correlation with clinical scores. Improvement in such correlation will be addressed in a future extension of the presented work.

\section{CONCLUSION}

In this paper, we presented a new framework to analyze time series images of the same subject for quantitative evaluation of subtle variations in facial wrinkles in response to a dermatological treatment. We proposed a registration scheme based on LDDMM and a wrinkle detection algorithm proposed previously by the first author. In future, the preliminary observations presented in this paper will be validated further by conducting experiments on larger dataset. In addition, since the performance of this framework depends greatly upon the accuracy of registration, we plan to improve registration scheme as well as validate our experimental results using clinical scores provided by the dermatologists.

\section{ACKNOWLEDGEMENT}

The authors would like to thank Marco Lorenzi and Yuliya Tarabalka (INRIA Sophia-Antipolis) for interesting discussions. The first author would also like to acknowledge the INRIA-DPE post doc fellowship. This research was conducted within the LIRA Consortium.

\section{REFERENCES}

[1] B. Klare, A.A. Paulino, and A.K. Jain, "Analysis of facial features in identical twins," in 2011 International 
Joint Conference on Biometrics (IJCB), Oct 2011, pp. $1-8$.

[2] G. O. Cula, P. R. Bargo, A. Nkengne, and N. Kollias, "Assessing facial wrinkles: automatic detection and quantification," Skin Research and Technology, vol. 19, no. 1, pp. e243-e251, 2013.

[3] G. O. Cula, P. R. Bargo, and N. Kollias, "Assessing facial wrinkles: automatic detection and quantification," 2009, vol. 7161, pp. 71610J-71610J-6.

[4] N. Batool and R. Chellappa, "Detection and Inpainting of Facial Wrinkles Using Texture Orientation Fields and Markov Random Field Modeling," IEEE Transactions on Image Processing, vol. 23, pp. 3773-3788, Sept. 2014.

[5] S.-G. Jeong, Y. Tarabalka, and J. Zerubia, "Marked point process model for facial wrinkle detection," in Proceedings of ICIP 2014, Oct. 2014.

[6] N. Batool and R. Chellappa, "Fast detection of facial wrinkles based on Gabor features using image morphology and geometric constraints," Pattern Recognition, vol. 48, no. 3, pp. $642-658,2015$.

[7] Z. Liu and J. Zerubia, "Towards automatic acne detection using a MRF model with chromophore descriptors," in Proceedings of the 21st European Signal Processing Conference (EUSIPCO),, Sept 2013, pp. 1-5.

[8] A. Maejima, A. Mizokawa, D. Kuwahara, and S. Morishima, "Facial aging simulation by patch-based texture synthesis with statistical wrinkle aging pattern model," in Mathematical Progress in Expressive Image Synthesis I, Ken Anjyo, Ed., vol. 4 of Mathematics for Industry, pp. 161-170. Springer Japan, 2014.

[9] J. Suo, X. Chen Chen, S. Shan, W. Gao, and Q. Dai, "A concatenational graph evolution aging model," IEEE Transactions on Pattern Analysis and Machine Intelligence, vol. 34, no. 11, pp. 2083-2096, 2012.

[10] T. Hund, B. Ascher, B. Rzany, and For the Smile Study Group, "Reproducibility of two four-point clinical severity scores for lateral canthal lines (crow's feet)," Dermatologic Surgery, vol. 32, no. 10, pp. 1256-1260, 2006.

[11] B. Ascher, B. Rzany, and R. Grover, "Efficacy and safety of botulinum toxin type a in the treatment of lateral crow's feet: Double-blind, placebo-controlled, dose-ranging study," Dermatologic Surgery, vol. 35, no. 10, pp. 1478-1486, 2009.

[12] A. Sotiras, C. Davatzikos, and N. Paragios, "Deformable medical image registration: A survey," IEEE
Trans. on Medical Imaging, vol. 32, no. 7, pp. 11531190, July 2013.

[13] M. F. Beg, M. I. Miller, A. Trouvé, and L. Younes, "Computing large deformation metric mappings via geodesic flows of diffeomorphisms," Int. J. Comput. Vision, vol. 61, no. 2, pp. 139-157, feb 2005.

[14] S.C. Joshi and M.I Miller, "Landmark matching via large deformation diffeomorphisms," IEEE Transactions on Image Processing, vol. 9, no. 8, pp. 1357-1370, Aug 2000.

[15] J. Glaunès, A. Qiu, M. I. Miller, and L. Younes, "Large deformation diffeomorphic metric curve mapping," International Journal of Computer Vision, vol. 80, no. 3, pp. 317-336, 2008.

[16] A. Qiu and M. I. Miller, "Cortical hemisphere registration via large deformation diffeomorphic metric curve mapping," Berlin, Heidelberg, 2007, MICCAI'07, pp. 186-193, Springer-Verlag.

[17] T. Vercauteren, X. Pennec, A. Perchant, and N. Ayache, "Diffeomorphic demons: Efficient non-parametric image registration," NeuroImage, vol. 45, no. 1, Supplement 1, pp. S61 - S72, 2009.

[18] M. Hernandez, S. Olmos, and X. Pennec, “Comparing algorithms for diffeomorphic registration: Stationary LDDMM and Diffeomorphic Demons," in 2nd MICCAI Workshop on Mathematical Foundations of Computational Anatomy, 2008, pp. 24-35.

[19] M. Hernandez, M. N. Bossa, and S.r Olmos, "Registration of anatomical images using paths of diffeomorphisms parameterized with stationary vector field flows," International Journal of Computer Vision, vol. 85, no. 3, pp. 291-306, 2009.

[20] Y. Fu, G. Guo, and T.S. Huang, "Age synthesis and estimation via faces: A survey," IEEE Transactions on Pattern Analysis and Machine Intelligence, vol. 32, no. 11, pp. 1955-1976, Nov 2010.

[21] N. Batool and R. Chellappa, "Modeling and detection of wrinkles in aging human faces using marked point processes," in Proceedings of the 12th International Conference on Computer Vision - Volume 2, Berlin, Heidelberg, 2012, ECCV'12, pp. 178-188, Springer-Verlag. 